\title{
Bürgerinteraktionen in der Smart City: Eine Kategorisierung
}

\author{
Tobias Tomczak/Kevin C. Andermatt/Kuno Schedler
}

Smart Cities nutzen moderne Informations- und Kommunikationstechnologien, womit neue Formen der Bürgerinteraktion möglich werden. In diesem Beitrag wird die Verwaltungsperspektive eingenommen und zwischen den Rollen der Bürger ${ }^{1}$ als Auftraggeber und als Kunden der Verwaltung unterschieden. Ersteres fokussiert sich auf die Steigerung der Legimitation und zweiteres auf die Steigerung des Kundennutzens. Außerdem werden die Interaktionsformen nach Art der Zusammenarbeit differenziert, indem zwischen Diagnose mit Fokus auf die Kreation und Operation mit Fokus auf die Leistung unterschieden wird. Vier unterschiedliche Interaktionstypen ergeben sich; Bürgerbeteiligung, demokratische Entscheidungsfindung, KoKreation und Ko-Produktion. Diese Kategorisierung hilft Smart Cities, Interaktionsformen zu entwickeln und erfolgreich umzusetzen.

\section{Einleitung}

Die Interaktion der Verwaltung mit ihren Bürgern ist ein altes Thema der Verwaltungswissenschaften. Wurde über längere Zeit die faktische Macht der Beamten beklagt und eine Machtbeschränkung durch die Politik gefordert, ${ }^{2}$ so steht spätestens

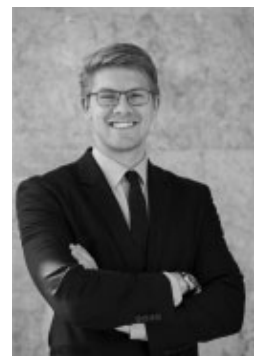

Tobias Tomczak

Doktorand im Bereich Public Management, Universität St. Gallen

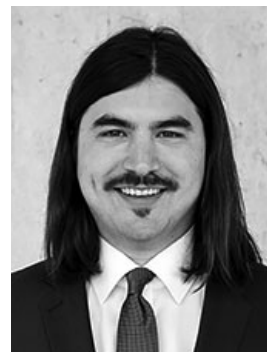

Kevin C. Andermatt

Assistent am Institut für VerwaltungsManagement, ZHAW seit dem New Public Management und dem E-Government der Einbezug der Bürger in die Entscheidungen ${ }^{3}$, die Innovationsprozesse $^{4}$ und die Leistungserstellung 5 der Verwaltung im Vordergrund.

Die Beteiligung von Bürgern am Verwaltungshandeln erhält im Zuge der Diskussionen rund um Smart City und Digitalisierung neuen Auftrieb. ${ }^{6}$ Dabei zeigt sich, dass insbesondere die europäischen Smart Cities einen starken Fokus auf neue, meist digitale Interaktionsformate legen. ${ }^{7}$ In den letzten Jahren hat sich ein Bewusstsein dafür entwickelt, dass die Entwicklung einer Smart City nicht einzig der technischen Rationalität untergeordnet werden darf. Eine rein technische

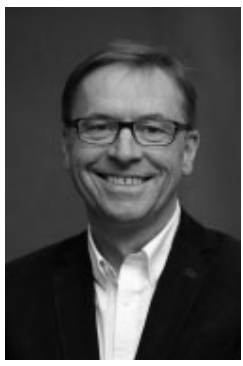

Kuno Schedler

Ordinarius für Public Management, Universität St. Gallen
Perspektive führt zu negativen externen Effekten hinsichtlich Datenschutz und Privatsphäre sowie gesellschaftlicher Inklusion und in Folge wird die Akzeptanz von Smart-City-Entwicklungen gemindert. ${ }^{8}$ Diese fehlende Akzeptanz zeigt sich insbesondere bei ostasiatischen Smart-CityInitiativen. So ist zum Beispiel das auf der „grünen Wiese“ entwickelte koreanische Smart-City-Projekt Songdo technisch mit allen Errungenschaften verwirklicht worden, dennoch wird die Stadt als leer empfunden. ${ }^{9}$ Ausgehend von diesen Erfahrungen werden heute in europäischen Städten partizipative und kooperative Gestaltungsansätze als erfolgsversprechender angesehen. Dank der Interaktion mit der Bevölkerung lässt sich eine ganzheitliche Betrachtung einer Smart City fördern und der Mensch rückt in Folge ins Zentrum der Stadtentwicklung.

Die Nähe zu den Bürgern steht seit vielen Jahren für eine Legitimität stiftende Form des Verwaltungshandelns und für einen vielversprechenden Weg, die Bedürfnisse der Bevölkerung besser identifizieren zu können, was die Entwicklung und Bereitstellung von nutzerzentrierten Leistun-

\footnotetext{
Aus Gründen der leichteren Lesbarkeit wird im Rahmen des vorliegenden Berichts auf eine geschlechtsspezifische Differenzierung - wie z.B. bei Bürger/Innen - verzichtet. Entsprechende Begriffe gelten im Sinne der Gleichbehandlung für beide Geschlechter.

Hellman 1918.

Bryson et al. 2013.

Callahan/Gilbert 2005.

Collm/Hristova/Schedler 2006

Bolívar/Muñoz 2019; Díaz-Díaz/Pérez-González 2016; Granier/Kudo 2016.

Nesti 2018

Joss/Cook/Dayot 2017; Joss et al. 2019.

Poon 2018
} 
gen forciert. ${ }^{10}$ So kann neben der Legitimität auch der Kundennutzen nachhaltig Verwaltung nimmt in einer Smart City an Bedeutung zu, da die Bürger einer Smart City als „pro-sumer"11 in die Erzielung von Wirkung staatlichen Handelns einbezogen werden.

Im deutschsprachigen Raum besteht bereits eine Fülle an Literatur und Konzepten zu Bürgerorientierung und -einbezug im allgemeinen Sinn. ${ }^{12}$ Was für die gesteigert werden. Diese Bürgernähe der de Interaktionskonzepte zu konsolidieren und auf die Smart City anzuwenden. Für die Verwaltungspraktiker wiederum zeigt der vorliegende Artikel auf, wie sich angesichts neuer technologischer Möglichkeiten und weiterhin knapper Ressourcen die passende Form der Bürgerinteraktion für ein möglichst effizientes und effektives Verwaltungshandeln finden lässt. Allgemein geht es darum, Bedürfnisorientierung und Nutzerzentrierung zu fördern und gleichzeitig die Legitimation für die dafür unternommenen Handlungen sowie
City wird als ein Mittel zur Bewältigung dieser Herausforderungen gesehen. ${ }^{13}$ Der Begriff „smart“ wird dabei unterschiedlich definiert. Während etwa von Lucke ${ }^{14}$ den Begriff in einem engeren Sinn vor allem mit dem Einsatz smarter Technologie („4.0“) verbindet, haben Gil-Garcia et al. ${ }^{15}$ „smart“ in einem sehr weiten Sinn als ein Feld mit 14 Dimensionen beschrieben; insbesondere relevant für diesen Beitrag sind Nutzerorientierung, Innovation, Kreativität, Effektivität und Bürgerengagement.

Das von Gil-Garcia et al. ${ }^{16}$ genannte Bürgerengagement betrifft dabei nicht nur die Bürger, sondern nicht zuletzt auch die Verwaltung einer Smart City. Sowohl aufgrund der gesellschaftlichen Entwicklung - insbesondere des erhöhten Bewusstseins für Nachhaltigkeit - als auch aufgrund der Versprechen des digitalen Wandels wird mehr Involvierung der Bürger gefordert. ${ }^{17}$ Bürger sollen generell von mehr Transparenz der Verwaltungen, mehr Mitspracherecht sowie kundenzentrierten Prozessen profitieren. Eine wichtige Rolle spielt hier die Privatwirtschaft, ${ }^{18}$ welche den Bürgern durch Unternehmen wie Amazon zeigt, wie komfortabel eine hohe Nutzerorientierung ist (z.B. Ein-KlickKauf). Der digitale Wandel ermöglicht einfachere Informationsbereitstellung und -beschaffung und ändert hierdurch die Interaktion mit den Bürgern. Dies wird an der zunehmenden Vernetzung von Menschen, Organisationen, Prozessen und Datenpunkten deutlich. Auf einer quantitativen Ebene können Bürgerumfragen einfach und kostengünstig durchgeführt werden und die so gewonnen Daten mit Hilfe von leistungsstarker und benutzerfreundlicher Software analysiert werden. Die daraus resultierenden Erkenntnisse wiederum ermöglichen die Entwicklung praktische Relevanz der Heuristik für die öffentliche Verwaltung einer Smart City dargelegt.

\section{Bürgerinteraktion in der Smart City}

Mit dieser Abgrenzung soll die Operationalisierung von Bürgerinteraktion im digitalen Zeitalter für die Wissenschaft erleichtert und die empirische Arbeit in diesem Feld durch einen Versuch der Systematisierung gestärkt werden. Dabei sollen nicht neue Begriffe eingeführt werden. Vielmehr geht es darum, bereits bestehen-
Angesichts zunehmender Urbanisierung stehen die Städte heute weltweit vor enormen Herausforderungen. Es gilt in einem sehr komplexen Umfeld, das Gemeinwohl sowie die wirtschaftliche Prosperität zu fördern und gleichzeitig eine nachhaltige Entwicklung zu gewährleisten. Die Smart

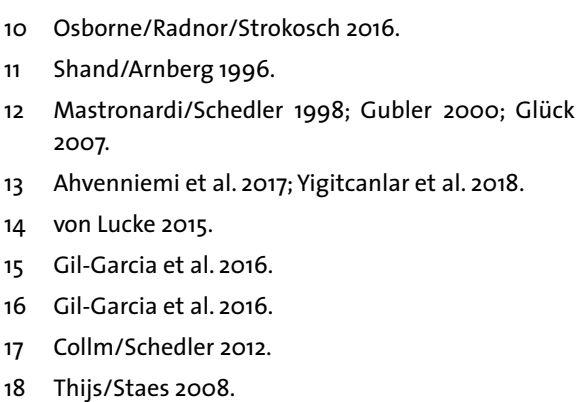


von effektiveren und effizienteren Leistungen für die diversen Anspruchsgruppen. Auf der qualitativen Ebene ergibt sich mehr Wertzuwachs durch die Involvierung der Bürger, da die Herausforderungen komplexer werden und ein tiefes Verständnis der Bedürfnisse der Bürger notwendig ist.

Dies führt zur folgenden Schlussfolgerung: Mit dem Aufkommen neuer Informations- und Kommunikationstechnologien, welche in einer Smart City zum Ein- konstitutiv für die politische Legitimation des Verwaltungshandelns. Die dahinterstehende Logik ist, dass die Bürger - etwas verkürzt - die Auftraggeber der Verwaltung sind.

Der Begriff Kooperation wird hingegen primär in der Public-ManagementDebatte verwendet und folgt eher einer betriebswirtschaftlichen Logik. Die Qualität der Leistungen der Verwaltung sowie die Erhöhung des Kundennutzens stehen dabei im Vordergrund. ${ }^{22}$ Indem die Bür-

\section{"Der Begriff Partizipation wird verwendet, wenn die Bürger einer Smart City in ihrer Rolle als Auftraggeber betrachtet werden. Wohingegen der Begriff Kooperation benutzt wird, wenn die Bürger die Rolle des Kunden einnehmen."}

satz kommen, wird die Partizipation von Bürgern und die Kooperation zwischen Bürgern und Verwaltung einen höheren Stellenwert erhalten.

Die Begriffe Partizipation und Kooperation werden sowohl in der Wissenschaft als auch in der Praxis häufig synonym verwendet oder unklar voneinander abgrenzt, ${ }^{19}$ was insbesondere im SmartCity-Kontext, wo die Begriffe von zentraler Bedeutung sind, zu Verständnisproblemen führen kann.

In der Tendenz kommt der Begriff „Partizipation“ aus der politikwissenschaftlichen Diskussion. Arnstein ${ }^{20}$ legt beispielsweise in ihrer Arbeit den Fokus auf die von der Gesellschaft vernachlässigten Bürger und untersucht, wie deren Bedürfnisse mittels mehr Bürgerpartizipation besser in die Politikgestaltung einfließen können. Folgerichtig werden bei der Partizipation die Bürger in ihrer Rolle des Souveräns angesprochen, ${ }^{21} \mathrm{der}$ auf generell-abstrakter Ebene politische Entscheidungen vorbereitet und fällt. Die Partizipation des Souveräns wirkt dabei ger in der Gestaltung und Umsetzung von Leistungsprozessen der Verwaltung mitwirken, sollen sie ihre individuell-konkreten Nutzenpotenziale besser ausschöpfen können. Die dahinterstehende Logik ist, dass die Bürger - wiederum verkürzt - die Kunden bzw. Konsumenten der Verwaltung sind. ${ }^{23}$

Ausgehend von diesen Überlegungen wird der Begriff Partizipation verwendet, wenn die Bürger einer Smart City in ihrer Rolle als Auftraggeber betrachtet werden. Wohingegen der Begriff Kooperation benutzt wird, wenn die Bürger die Rolle des Kunden einnehmen.

Neben den Rollenverständnissen gilt es aber auch die verschiedenen Phasen des Verwaltungsprozesses zu berücksichtigen. Hier bietet sich eine Unterscheidung zwischen den Kernprozessen „Diagnose $\Rightarrow$ Kreation“ und „Operation $\Rightarrow$ Leistung“ an.

Beim Prozess „Diagnose $\Rightarrow$ Kreation“ ist die Art der Interaktion typischerweise informell und die Art des Inputs der Bür- ger ist definiert. Die Bürger bringen ihr Wissen und ihre Zeit als Input in den Prozess ein. Hierfür eignen sich insbesondere digitale Medien wie Social Media, wo die Bürger bereits aktiv sind und mit wenig Aufwand ihren Input erbringen können. Dies ist ein ergebnisoffener Prozess, in dem sich nicht vorgängig definieren lässt, welche Lösungen gefunden werden. Weiter weist bei diesem Kernprozess die Interaktion einen informellen Charakter auf, d.h. es besteht kein Vertragsverhältnis zwischen den involvierten Parteien. Vielmehr handelt es sich um einen losen Zusammenschluss, der sich meist spontan ergibt und keine definierten Leistungen und Gegenleistungen vorsieht. ${ }^{24}$ Die aus einem informalen Interaktionsprozess resultierenden Entscheidungen sind rechtlich nicht bindend. Die Generierung von Wissen und damit die Stärkung der Innovationskraft stehen im Vordergrund.

Beim Prozess „Operation $\Rightarrow$ Leistung“ ist die Art der Interaktion hingegen formal ausgestaltet und aus Prozessperspektive ist die Art des Ergebnisses vorgängig definiert. Die Anspruchsgruppen erhalten eine definierte Leistung (z.B. Erhalt einer elektronischen Patientenakte bei Lieferung von Krankenakten) im Hinblick darauf, dass eine bestimmte Wirkung erzielt werden soll. Digitale Anwendungen sind hierfür prädestiniert, wenn sie die standardisierte Aufnahme von Informationen effektiv und effizient ermöglichen. Bei einer solchen Form der Interaktion besteht der Zusatznutzen insbesondere in datenbasierten (und damit informierten) Entscheidungen sowie in den zusätzlichen Erbringungs- und Verwaltungskapazitäten für die beteiligten Bürger. Außerdem wird bei einer solchen formalen Interaktion von einer klar definierten Leistung und Gegenleistung über einen bestimmten Zeitraum ausgegangen. In der Regel besteht zwischen den involvierten Parteien ein Vertragsverhältnis, und die Interaktion

\footnotetext{
19 z.B. Lee-Geiller/Lee 2019

20 Arnstein 1969.

21 Oser/Hooghe/Marien 2013; Jacobs/Kaufmann 2019.

22 Osborne/Strokosch 2013; Osborne/Radnor/ Strokosch 2016.

23 Osborne/Radnor/Strokosch 2016; Nabatchi/ Sancino/Sicilia 2017.

24 Collm/Schedler 2012.
} 
basiert auf einer entsprechenden rechtlichen Grundlage.

Im Kernprozess „Diagnose $\Rightarrow$ Kreation “ sind also die Beiträge tendenziell inputbezogen und informal. Die Innovationskapazität der Verwaltung steht dabei im Vordergrund. Hingegen ist der Kernprozess „Operation $\Rightarrow$ Leistung“ tendenziell outputbezogen und formalisiert. Im Vordergrund steht hier die Erbringungskapazität der Verwaltung.

Zusammenfassend lässt sich festhalten, dass der technologische Wandel und das Aufkommen von Smart Cities die Partizipation von Bürgern und die Kooperation zwischen Bürgern und Verwaltung maßgeblich verändern wird. Für eine operationalisierbare Abgrenzung und generell für ein besseres Verständnis lohnt es sich, den Begriff Partizipation mit der Rolle des Bürgers als Auftraggeber sowie den Begriff Kooperation mit der Rolle des Bürgers als Kunden zu komplementieren. Schließlich gilt es zu beachten, dass bei Betrachtung der beiden Kernprozesse „Diagnose $\Rightarrow$ Kreation“ und „Operation $\Rightarrow$ Leistung “ sich die prozesseigenen Interaktionsformen zu unterscheiden haben. Aus diesen Überlegungen lässt sich die in Abbildung 1 dargestellte Heuristik mit vier Formen der Bürgerinteraktion ableiten.

\section{Die vier Formen der Bürger- interaktion in einer Smart City}

Nachfolgend sollen die in der Heuristik definierten Formen der Bürgerinteraktion im Kontext von Smart Government (Verwaltung einer Smart City) näher beschrieben werden.

\section{Bürgerbeteiligung}

Unter dem Begriff Bürgerbeteiligung wird die kreative gemeinsame Entwicklung eines Outputs für die Gemeinde in einem offenen Prozess verstanden. Die Bürger werden von der öffentlichen Verwaltung dazu eingeladen, die Gemeinde mit zu entwickeln. Der Input besteht dabei aus den Kognitionen der Teilnehmenden sowie deren aufgewendete Zeit und erfolgt in digitaler oder analoger Form. Der Output steht jedoch vorgängig noch nicht fest. Bürgerbeteiligung zielt darauf ab, Bürger als Souverän vermehrt in die Gestaltung

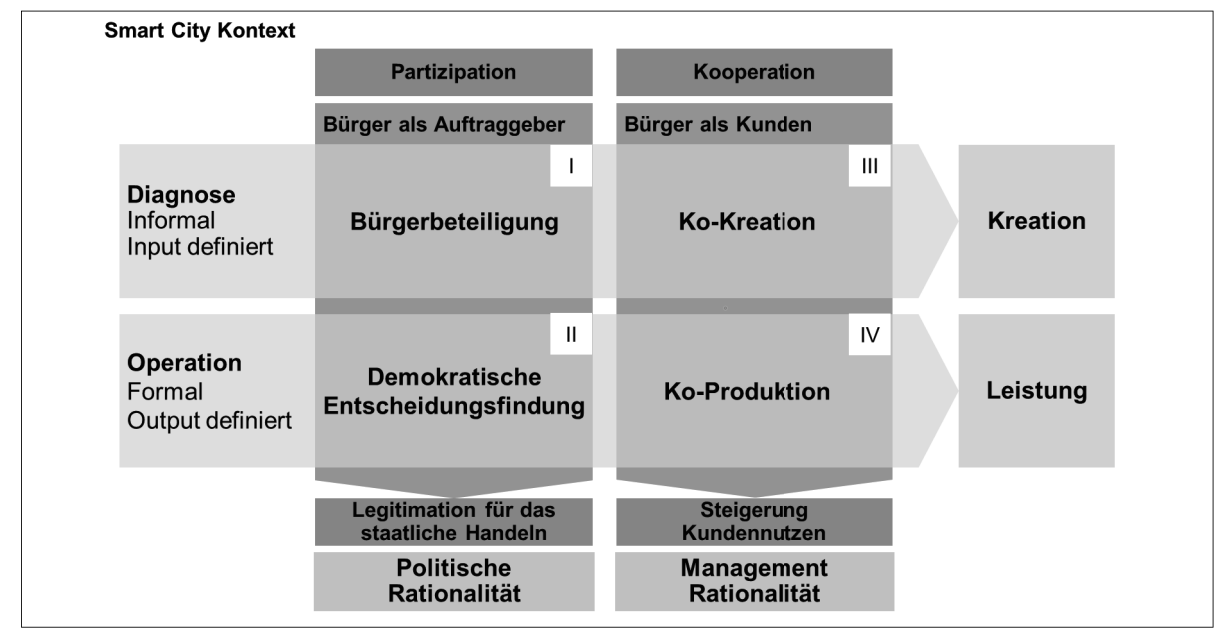

Abb. 1: Die vier Formen der Interaktion

der Gemeindeentwicklung einzubeziehen und in Folge die politische Legitimation zu steigern. E-Partizipation wird die Bürgerbeteiligung im digitalen Raum genannt. ${ }^{25}$ Hierfür eignen sich Webseiten und soziale Plattformen wie Facebook und Instagram. Städte können über diese Instrumente die Bevölkerung informieren und mit ihr in einen ständigen Austausch treten. So existiert die Webseite „Vorarlberg Mitdenken“, ${ }^{26}$ bei welcher die Vorarlberger Gemeinden sich mit ihrer Bevölkerung austauschen können. Beispielweise nutzte die Stadt Bregenz die Plattform für die Initiative „Selbstverständnis Bregenz“, welche die kreative gemeinsame Weiterentwicklung der Stadt zum Ziel hatte. Grundsätzlich ist die Beteiligung der Bevölkerung im digitalen Raum kostengünstig, besitzt jedoch den Nachteil, nur eine begrenzt vertiefte Diskussion zu ermöglichen. ${ }^{27}$ So hat die Stadt Bregenz auch auf den analogen Austausch gesetzt und befragte die Bürger auch in den Fußgängerzonen. Dies ermöglichte ein tiefgehendes Verständnis für die Bürger und ihrer Ansichten. Der analoge Austausch mit der Bevölkerung - die klassische Bürgerbeteiligung - ist und bleibt wichtig für komplexe und nicht greifbare Themen wie die Entwicklung einer „Smart City Strategie“.

\section{Fallbeispiel: Bürgerbeteiligung Entwicklung Smart City Strategie ${ }^{28}$}

Im Rahmen der \#smarthalle der Stadt St. Gallen wurde die Bevölkerung zu zwei Workshops eingeladen, um die Smart City-Strategie der Stadt mit zu entwickeln. Die Stadt St. Gallen eröffnete für 70 Tage einen Pop-up Store (die \#smarthalle), um den Bürgern die Smart City „erlebbar“ zu machen. Das sehr abstrakte Konzept der Smart City konnte auf diesem Weg den Bürgern nähergebracht werden, damit die Verwaltung die Legitimität und Akzep$\operatorname{tanz}$ für weitere Investitionen in die $\mathrm{Zu}$ kunft der Stadt St. Gallen erhält. In zwei Workshops, die einen wertvollen Teil des Smarthalle-Projektes darstellten, konnte die Bevölkerung ihre Bedürfnisse, Wünsche und Ideen für die smarte Stadt St. Gallen formulieren. Hierfür konnten sie mit Mitarbeitern der Verwaltung und Experten diskutieren. Dies ermöglichte der Verwaltung, auf die von der Bevölkerung wahrgenommenen Chancen und Risiken einzugehen.

\section{Demokratische Entscheidungsfindung}

Unter demokratischer Entscheidungsfindung werden institutionalisierte politische Prozesse der Beschlussfassung verstanden. Konkret können dies parlamentarische Beschlüsse, Exekutiventscheidungen oder in der Schweiz auch Referenden oder Volksinitiativen sein. Der Output ist jeweils eine demokratisch legitimierte Entscheidung, und diese Entscheidung ist in Form einer Rechtsnorm klar definiert. Diese Form der Interaktion liefert somit legitimierte Rechtsgrundlagen, die

\footnotetext{
25 Wirtz/Daiser/Binkowska 2018.

26 «Vorarlberg Mitdenken» Plattform 2020.

27 Androutsopoulou et al. 2019.

28 Stadt St. Gallen 2019.
} 
für das Verwaltungshandeln maßgeblich sind und unterscheidet sich somit von der Bürgerbeteiligung in ihrer Bindungskraft. Mit der fortschreitenden Digitalisierung werden solche demokratischen Entscheidungsprozesse auch digital möglich. In diesem Kontext wird oft von „Digitaler Demokratie“ oder „E-Democracy“ gesprochen. ${ }^{29}$ Im Grunde geht es darum, mit Hilfe digitaler Kanäle die demokratischen Prozesse für die jeweiligen Anspruchsgruppen zugänglicher zu machen bzw. für die breite Bevölkerung zu öffnen. Die digitale Demokratie ist für eine Smart City von zentraler Bedeutung, denn eine smarte Stadt möchte die Bevölkerung stärker in die politische Entscheidungsfindung verknüpft, dass sich über den digitalen Kanal mehr Menschen an der demokratischen Entscheidungsfindung beteiligen. ${ }^{34}$ Die Kritiker von E-Voting wiederum befürchten, dass bei einer (frühzeitigen) Einführung der digitalen Stimmkanals die Demokratie unnötig gefährdet wird. Konkret wird befürchtet, dass das digitale System „gehackt“" werden könnte. ${ }^{35}$

\section{Fallbeispiel: E-Voting in der Schweiz}

Aufgrund der direkt-demokratischen Tradition werden in der Schweiz Stimmbürger mehrmals jährlich zur Wahl- bzw. Abstimmungsurne gebeten. Im Zuge der Digitalisierung wurden in den letzten Jah-

\section{"Ko-Kreation ermöglicht eine hohe Nutzerzentrierung der digitalen Leistungen, was wiederum eine Smart City speziell auszeichnet."}

einbinden. Dadurch wird eine höhere Akzeptanz bzw. Legitimität der Stadtentwicklung angestrebt. ${ }^{30}$

In der Schweiz haben Initiativen im Bereich „Digitaler Demokratie“ meist zum Ziel, die direkte Demokratie zu stärken. ${ }^{31}$ Beispielsweise soll durch das so genannte E-Collecting das Sammeln von Unterschriften für Referenden oder Volksinitiativen auch elektronisch möglich werden. Im Kanton Schaffhausen wird momentan geprüft, ob sich E-Collecting - digitalisierte Unterschriftensammlung - auf kantonaler Ebene einführen lässt. Die dazu benötigte elektronische Identität besitzt der Kanton Schaffhausen bereits. ${ }^{32}$ Der Klassiker der digitalen Demokratie ist aber das viel diskutierte und gerade in der Schweiz auch stark umstrittene „E-Voting“. Mit Hilfe von E-Voting soll die Wahl der Volksvertreter auch auf digitalem Weg ermöglicht werden. In der Schweiz wäre mit E-Voting außerdem die elektronische Stimmabgabe bei Volksbegehren möglich. ${ }^{33}$ Mit E-Voting wird die Hoffnung ren in verschiedenen Kantonen Projekte gestartet, die die Einführung eines elektronischen Stimmkanals zum Ziel hatten. ${ }^{36}$ E-Voting steht dafür, dass die Stimmbürgerinnen und Stimmbürger übers Internet abstimmen und wählen können. So soll eine orts- und zeitunabhängige Stimmabgabe ermöglicht werden. Die Eidgenössische Bundeskanzlei, die sich stark für das E-Voting einsetzt, erhofft sich durch den digitalen Kanal eine Revitalisierung der Stimmbeteiligung in der Schweiz. ${ }^{37}$ In den Jahren 2018 und 2019 haben aber alle Kantone, die sich mit der Einführung von E-Voting beschäftigten, ihre Versuchsbetriebe eingestellt. Der Grund für den Stopp basierte meist auf Sicherheitsbedenken. So stellte der Kanton Genf im 2019 sein eigenes E-Voting-System ein und auch die Post musste ihr E-Voting-System aus Sicherheitsgründen zurückziehen. Folglich existiert in der Schweiz momentan kein EVoting. Die Schweizerische Post hat aber für 2020 ein neues System angekündigt. ${ }^{38}$ Gleichzeitig läuft jedoch die Unterschriftensammlung für ein E-Voting-Moratori- um. Eine Volksinitiative, die E-Voting in der Schweiz faktisch verbieten würde. ${ }^{39}$

\section{Ko-Kreation}

Die digitale Transformation ermöglicht ganz neue Verwaltungsleistungen. Eine optimale Ausgestaltung dieser Leistungen wird durch eine frühe Einbeziehung der Nutzer in den Entwicklungsprozess befördert. ${ }^{40}$ Diese Form der Ko-Kreation ermöglicht eine hohe Nutzerzentrierung der digitalen Leistungen, was wiederum eine Smart City speziell auszeichnet. Unter dem Begriff Ko-Kreation wird die kreative gemeinsame Entwicklung eines Outputs in einem offenen Prozess verstanden. ${ }^{41}$ Die Bürger, die in dieser Form der Interaktion als Kunden angesprochen werden, werden von der öffentlichen Verwaltung dazu eingeladen, die Leistungen mit zu entwickeln und an innovationsfördernden Prozessen zu partizipieren. ${ }^{42}$ Beispielsweise werden Workshops zu neuen Service-DeliveryModellen veranstaltet. Hierfür werden mit den Kunden kooperative Design-Verfahren (z.B. Design-Thinking) durchlaufen. Dies kann zum Beispiel in Form von Workshops oder auf dem Weg der Analyse von sogenannten Customer-Journeys geschehen. ${ }^{43}$ Dank neuen Technologien können in einer Smart City die Bürger bzw. Kunden noch stärker in die Leistungsentwicklung einbezogen werden. ${ }^{44}$ Mit Hilfe von stadteigenen Apps können die Kunden zeit- und ortsunabhängig Rückmeldungen zu den Verwaltungsleistungen geben. Durch Bewertungssysteme, die in die Verwaltungswebsites integriert werden, können laufend Verbesserungsvorschlä-

\footnotetext{
29 Lee/Chang/Berry 2011.

o Michels/De Graaf 2010.

Ammann/Schnell 2019.

Kanton Schaffhausen 2019.

Ammann/Schnell 2019.

Bundeskanzlei BK 2020.

Schäfer 2019

E-Government Schweiz 2020.

Bundeskanzlei BK 2020.

E-Government Schweiz 2020.

Schäfer 2019.

Galvagno/Dalli 2014.

Grönroos 2011; Osborne 2018

Collm/Schedler 2012.

Stickdorn et al. 2016.

z.B. Johnson/Robinson/Philpot 2019.
} 
ge eingebracht werden und mit Hilfe von Online-Umfragen lassen sich gezielt die Einschätzungen von spezifischen Leistungen und Prozessen der Smart City einholen. ${ }^{45}$ Die Verwendung digitaler Lösungen unterstützt offene Innovationsprozesse (Open Innovation), die einen ganzheitlichen Blick auf das Verwaltungshandeln der Smart City und eine laufende Optimierung der Leistungen ermöglichen.

\section{Fallbeispiel: Ko-Kreation der Schaffhauser eID+}

Bereits früh hat der Kanton Schaffhausen auf die digitale Transformation der eigenen Verwaltung gesetzt. Schaffhausen war auch der erste Schweizer Kanton, der seinen Bürgern eine elektronische Identität anbot. Bei der Entwicklung der Schaffhauser eID+ wurden die Bevölkerung sowie die zuständigen Verwaltungsstellen aktiv einbezogen. Während der Pilotphase wurde in Kooperation mit einem Forschungsinstitut die Beta-Version der Schaffhauser eID+ mittels Ko-Kreation bzw. Ko-Design optimiert. ${ }^{46}$ Die Bevölkerung wurde dazu eingeladen, an einem Usability-Test mit anschließender Befragung teilzunehmen. Dabei wurde die Front-End-Version der Schaffhauser eID+ getestet und zusammen mit der Bevölkerung wurde eruiert, wie sich die Applikation noch stärker an den Bedürfnissen der Nutzer ausrichten lässt. Gleichzeitig wurde auch das Back-End der Applikation zusammen mit der zuständigen Verwaltungsstelle getestet. So konnte in Interaktion mit den Bürgern bzw. den potenziellen Kunden der Leistung eine Nutzerzentrierung der eID-Applikation erarbeitet werden. Die Schaffhauser eID+ wird von der Bevölkerung angenommen und wird bereits von rund 1.000 Einwohnern genutzt. $^{47}$

\section{Ko-Produktion}

Bei der Ko-Produktion stehen die gemeinsame Erschaffung und Lieferung in einem festgelegten und formalen Prozess im Vordergrund. ${ }^{48}$ Die Kunden interagieren mit der Verwaltung, um ihren Beitrag an die Erzielung einer Wirkung zu leisten. Die Kunden „bezahlen“ nicht nur für eine Leistung, sondern bringen sich aktiv in den Produktionsprozess ein. Sie sind also „pro-sumers“.49 Das Ziel dieser Interakti- on besteht darin, die Kunden einzubinden, um die Erbringung der Leistung überhaupt zu ermöglichen. Durch die Digitalisierung können die Bürger noch stärker in die Leistungserbringung einbezogen werden. Die Digitalisierung ermöglicht einen viel intensiveren Austausch von Informationen. So liefern die Bürger Informationen, welche es den Verwaltungen ermöglichen, neue Service-Delivery-Modelle einzuführen und generell effizienter sowie effektiver ihre Services anzubieten. Die Digitalisierung ermöglicht es, die Informationen einfacher und kostengünstiger zu liefern. So können Bürger über Bürgermeldeplatt- und können mit Erlaubnis des Patienten abgefragt werden. Richtig umgesetzt ist das System für die Patienten effizienter, effektiver und sicherer. Ein Beispiel ist das MonDossierMedical.ch aus Genf mit bereits über 46.000 registrierten Patienten. ${ }^{51}$

\section{Relevanz für die Verwaltung}

$\mathrm{Zu}$ Beginn des digitalen Wandels in der Verwaltung und Politik standen noch die neuen Technologien im Vordergrund. ${ }^{52}$ Mehr und mehr wird nun der Mensch in den Mittelpunkt der Überlegungen gerückt. So werden zurzeit viele Smart-

\section{"Zu Beginn des digitalen Wandels in der Verwaltung und Politik standen noch die neuen Technologien im Vordergrund. Mehr und mehr wird nun der Mensch in den Mittelpunkt der Überlegungen gerückt.»}

formen auf Straßenschäden hinweisen und koproduzieren die Leistung „funktionierende Infrastruktur “. ${ }^{50}$ Bürger können eine eID nutzen und so die Erbringung von Serviceleistungen privat wie öffentlich vereinfachen. Sie können es der Verwaltung erlauben, persönliche Informationen auf einem Bürgerkonto zu speichern und ermöglicht der Verwaltung so, vorausgefüllte Formulare zu verschicken und proaktiv auf die Bürger zuzugehen (z.B. wenn der Pass abläuft).

\section{Fallbeispiel: Ko-Produktion der ePatien- tendossiers}

Ein ePatientendossier beinhaltet die medizinischen Informationen über einen Patienten in elektronischer Form. Die Nutzer willigen ein, ihre medizinischen Informationen zu teilen. Hiermit tragen sie aktiv zum Produktionsprozess der Leistung bei. Der Prozess ist klar formalisiert. Es bestehen feste Regeln, wie der Vorgang des Datenteilens erfolgt. Zudem ist auch der Output klar definiert. Die medizinischen Informationen befinden sich an einem Ort
City- und Smart-Government-Strategien veröffentlicht, welche die Interaktion mit den Bürgern als einen Hauptbestandteil der zukünftigen Entwicklung betrachten. ${ }^{53}$ Der vorliegende Artikel verdichtet die verschiedenen Formen der Bürgerinteraktion auf vier generische Typen und entwickelt auf dieser Basis eine handlungsleitende Kategorisierung für Wissenschaft und Praxis. Die vier vorgestellten Formen ermöglichen eine praktikable Abgrenzung und streichen hierdurch die jeweils situativ unterschiedlichen Herausforderungen klar heraus. Daraus lassen sich wiederum passende Strategien und Aktionen für die Entwicklung von Smart Cities ableiten.

\footnotetext{
45 z.B. Wirtz/Daiser/Binkowska 2018.

46 Andermatt/Göldi 2018.

47 Kanton Schaffhausen 2019.

48 Osborne 2018.

49 Shand/Arnberg 1996.

50 z.B. Stadt Bregenz 2020.

51 Etat Genève 2018.

52 von Lucke 2015.

53 z.B. Stadt Zürich 2020.
} 
Die hier vorgestellten vier Formen der Bürgerinteraktion beschäftigen sich grundlegend mit der Art und Weise, wie Bürger in differenzierter Form mit der Politik und der Verwaltung interagieren. Das vorgestellte Modell liefert eine Basis für vertiefende Analysen. So können in der Folge beispielsweise Fragen nach der Art der geeigneten Kommunikationen mit den Bürgern situativ beantwortet werden. Generell ist zu beachten, dass mehr Interaktion nicht zwingend zu besseren Leistungen seitens der Verwaltung führt. Je nach Thematik fehlt es den Bürgern an Expertise und Interesse, sich vertieft einzubringen. ${ }^{54}$ Umso wichtiger ist es deshalb, die verschiedenen Interaktionsformen anhand ihrer Eigenschaften einzuordnen, um so je nach Thema entscheiden zu können, ob und falls ja, welche Bürgerinteraktion zielführend sein könnte. Schließlich gilt auch heute die Restriktion knapper Ressourcen.

Je nachdem, in welche Kategorie eine Interaktion fällt, muss sich die Verwaltung außerdem unterschiedlich auf den Prozess vorbereiten. Die Anforderungen an eine formelle, demokratisch legitimierte Entscheidungsfindung sind beispielsweise deutlich höher als an eine Involvierung der Bürger in das Neu-Design eines Verwaltungsprozesses. Das Bewusstsein für die benötigte Art der Bürgerinteraktion kann vor allem für die Verwaltung entlastend wirken, da ein Großteil der Innovationen im Zusammenhang mit Smart Government tiefere formelle Anforderungen mit sich bringen.

54 Buckwalter 2014; lanniello et al. 2019.

\section{Literatur}

Ahvenniemi, H./Huovila, A./Pinto-Seppä, I./ Airaksinen, M. (2017): What are the differences between sustainable and smart cities?, in: Cities Nr. 6o, S. 234-245.

Ammann, M./Schnell, F. (2019): Digitale Direkte Demokratie. Verfügbar unter: https://www. avenir-suisse.ch/publication/digitale-direktedemokratie/.

Andermatt, K.C./Göldi, R.A. (2018): Introducing an Electronic Identity: The Co-design Approach in the Canton of Schaffhausen, in: Jahrbuch der Schweizerischen Verwaltungswissenschaften Nr. 9(1).

Androutsopoulou, A./Karacapilidis, N./Loukis, E./Charalabidis, Y. (2019): Transforming the communication between citizens and government through Al-guided chatbots, in: Government Information Quarterly Nr. 36(2), S. 358-367.

Arnstein, S.R. (1969): A Ladder Of Citizen Participation, in: Journal of the American Institute of Planners Nr. 35(4), S. 216-224.

Bryson, J.M./Quick, K.S./Slotterback, C.S./Crosby, B.C. (2013): Designing public participation processes, in: Public administration review Nr. 73(1), S. 23-34.

Buckwalter, N.D. (2014): The Potential for Public Empowerment through Government-Organized Participation, in: Public Administration Review Nr. 74(5), S. 573-584.

Bundeskanzlei BK (2020): Vote électronique. Verfügbar unter: https://www.bk.admin.ch/ bk/de/home/politische-rechte/e-voting.html (Zugegriffen: 22. März 2020).

Callahan, R.F./Gilbert, G.R. (2005): End-User Satisfaction and Design Features of Public Agencies, in: The American Review of Public Administration Nr. 35(1), S. 57-73.

Collm, A./Hristova, R./Schedler, K. (2006): E-Collaboration in der öffentlichen Verwaltung in der Schweiz.

Collm, A./Schedler, K. (2012): Managing crowd innovation in public administration, in: International Public Management Review Nr. 13(2), S. 1-18.

Díaz-Díaz, R./Pérez-González, D. (2016): Implementation of social media concepts for e-government: Case study of a social media tool for value co-creation and citizen participation, in: Journal of Organizational and End User Computing Nr. 28(3), S. 104-121.

E-Government Schweiz (2020): E-Voting neu ausrichten und stabilen Versuchsbetrieb sicherstellen - www.egovernment.ch. Verfügbar unter: https://www.egovernment.ch/de/umsetzung/schwerpunktplan/vote-electronique/ (Zugegriffen: 22. März 2020).

Etat Genève (2018): MonDossierMedical.ch Connecté à ma santé, Département de l'emploi et de la santé. Verfügbar unter: http://www. mondossiermedical.ch/ (Zugegriffen: 22. März 2020).

Galvagno, M./Dalli, D. (2014): Theory of value co-creation: a systematic literature review, in: Managing Service Quality: An International Journal.

Gil-Garcia, J.R./Zhang, J./Puron-Cid, G. (2016): Conceptualizing smartness in government: An integrative and multi-dimensional view, in: Government Information Quarterly Nr. 33(3), S. 524-534.
Glück, M. (2007): Vertrauen und Legitimation durch Bürgerzufriedenheit: eine Untersuchung der Aufgabenerfüllungszufriedenheit anhand deutscher Städte und Gemeinden. Haupt Verlag AG.

Granier, B./Kudo, H. (2016): How are citizens involved in smart cities? Analysing citizen participation in Japanese "Smart Communities", in: Information Polity Nr. 21(1), S. 61-76.

Grönroos, C. (2011): Value co-creation in service logic: A critical analysis, in: Marketing Theory Nr. 11(3), S. 279-301.

Gubler, E. (2000): Von der Produktions- zur Markt- und Kundenorientierung, in Hablützel, P./Letsch, B. (Hrsg.): Vom Service Public zum Service au Public. Zürich: Neue Zürcher Zeitung, S. $175-181$.

Hellman, S. (1918): Die innere Politik. Herausgegeben von S. Hellman. Frankfurt.

lanniello, M./lacuzzi, S./Fedele, P./Brusati, L. (2019): Obstacles and solutions on the ladder of citizen participation: a systematic review, in: Public Management Review. Routledge Nr. 21(1), S. 21-46.

Jacobs, D./Kaufmann, W. (2019): The right kind of participation? The effect of a deliberative mini-public on the perceived legitimacy of public decision-making, in: Public Management Review Nr. S. 1-21.

Johnson, P.A./Robinson, P.J./Philpot, S. (2019): Type, tweet, tap, and pass: How smart city technology is creating a transactional citizen, in: Government Information Quarterly.

Joss, S./Sengers, F./Schraven, D./Caprotti, F./ Dayot, Y. (2019): The smart city as global discourse: Storylines and critical junctures across 27 cities, in: Journal of Urban Technology Nr. 26(1), S. 3-34.

Joss, S./Cook, M./Dayot, Y. (2017): Smart cities: Towards a new citizenship regime? A discourse analysis of the British smart city standard, in: Journal of Urban Technology Nr. 24(4), S. 29-49.

Kanton Schaffhausen (2019): Schaffhauser elD+ - Kanton Schaffhausen. Verfügbar unter: https://sh.ch/CMS/Webseite/KantonSchaffhausen/Beh-rde/Services/SchaffhauserelD--2077281-DE.html (Zugegriffen: 22. März 2020).

Lee-Geiller, S./Lee, T. (David): (2019): Using government websites to enhance democratic E-governance: A conceptual model for evaluation, in: Government Information Quarterly Nr. 36(2), S. 208-225.

Lee, C./Chang, K./Berry, F.S. (2011): Testing the development and diffusion of e-government and e-democracy: A global perspective, in: Public Administration Review Nr. 71(3), S. 444-454.

von Lucke, J. (2015): Smart Government-Wie uns die intelligente Vernetzung zum Leitbild „Verwaltung 4.0" und einem smarten Regierungsund Verwaltungshandeln führt.

Mastronardi, P./Schedler, K. (1998): New public management in Staat und Recht: ein Diskurs. Haupt.

Michels, A./De Graaf, L. (2010): Examining citizen participation: Local participatory policy making and democracy, in Local Government Studies Nr. 36(4), S. 477-491.

Nabatchi, T./Sancino, A./Sicilia, M. (2017): Varieties of Participation in Public Services: The Who, 
When, and What of Coproduction, in: Public Administration Review Nr. 77(5), S. 766-776.

Nesti, G. (2018): Defining and assessing the transformational nature of smart city governance: Insights from four European cases, in: International Review of Administrative Sciences.

Osborne, S. P. (2018): From public service-dominant logic to public service logic: are public service organizations capable of co-production and value co-creation?, in: Public Management Review Nr. 20(2), S. 225-231.

Osborne, S. P./Radnor, Z./Strokosch, K. (2016): Co-Production and the Co-Creation of Value in Public Services: A suitable case for treatment?, in: Public Management Review Nr. 18(5), S. 639653.

Osborne, S. P./Strokosch, K. (2013): It takes Two to Tango? Understanding the Co-production of Public Services by Integrating the Services Management and Public Administration Perspectives, in: British Journal of Management Nr. 24, S. 31-47.

Oser, J./Hooghe, M./Marien, S. (2013): Is Online Participation Distinct from Offline Participation? A Latent Class Analysis of Participation Types and Their Stratification, in: Political Research Quarterly Nr. 66(1), S. 91-101.

Poon, L. (2018): Sleepy in Songdo, Korea's Smartest City. Verfügbar unter: https://www. citylab.com/life/2018/o6/sleepy-in-songdo-koreas-smartest-city/561374/ (Zugegriffen: 20. März 2020).
Bolivar, M.P.R./Muñoz, L.A. (2019): E-participation in smart cities: technologies and models of governance for citizen engagement, Taylor \& Francis.

Schäfer, F. (2019): Breite Allianz verlangt Moratorium für E-Voting, Neue Zürcher Zeitung. Verfügbar unter: https://www.nzz.ch/schweiz/ breite-allianz-verlangt-moratorium-fuer-e-voting-Id.1454472.

Shand, D./Arnberg, M. (1996): The role of client choice in improving public sector performance, in OECD (Hrsg.): Responsive Government: Service Quality Initiatives. Paris: OECD.

Stadt Bregenz (2020): Bregenz - I LUAG UF DI. Verfügbar unter: https://www.iluagufdi.bregenz.at/home (Zugegriffen: 22. März 2020).

Stadt St. Gallen (2019): Stadt St.Gallen mobile | \#smarthalle. Verfügbar unter: https://m.stadt. sg.ch/index.php?apid=1985337896\&apparentid=16346254 (Zugegriffen: 22. März 2020).

Stadt Zürich (2020): Strategie Smart City Zürich - Stadt Zürich. Verfügbar unter: https://www. stadt-zuerich.ch/prd/de/index/stadtentwicklung/smart-city.html (Zugegriffen: 22. März 2020).

Stickdorn, M./Hormess, M. E./Lawrence, A./ Schneider, J. (2016): This is service design doing: Using research and customer journey maps to create successful services. O'Reilly Media.

Thijs, N./Staes, P. (2008): European Primer on Customer satisfaction management, European Quality Conference.
«Vorarlberg Mitdenken» Plattform (2020): "Vorarlberg Mitdenken" Plattform | Land Vorarlberg (Kooperationsprojekt). Verfügbar unter: https://vorarlberg.mitdenken.online/ (Zugegriffen: 22. März 2020).

Wirtz, B. W./Daiser, P./Binkowska, B. (2018): E-participation: A Strategic Framework, in: International Journal of Public Administration Nr. 41(1), S. 1-12.

Yigitcanlar, T./Kamruzzaman, M./Buys, L./loppolo, G./Sabatini-Marques, J./da Costa, E. M./ Yun, J. H. J. (2018): Understanding 'smart cities': Intertwining development drivers with desired outcomes in a multidimensional framework, in: Cities Nr. 81, S. 145-160.

\section{"Ein Lehrbuch, das viel bietet und einem wirklich weiterhilft."}

Studium 105/2019, 31, zur Vorauflage

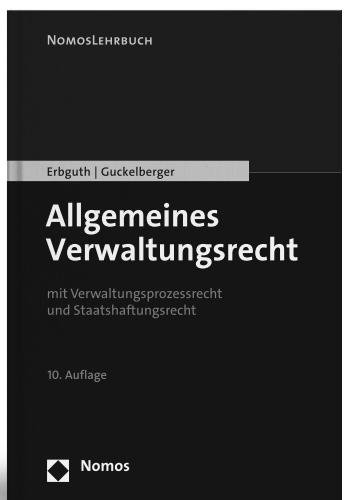

\author{
Allgemeines Verwaltungsrecht \\ mit Verwaltungsprozessrecht und Staatshaftungsrecht \\ Von Prof. Dr. Wilfried Erbguth und Prof. Dr. Annette Guckelberger \\ 10. Auflage 2020, 666 S., brosch., 25,90€ \\ ISBN 978-3-8487-6097-8
}

Das Lehrbuch wurde für die 10. Auflage grundlegend überarbeitet und ist aufStand Juni 2019. Einbezogen wurden u.a. die jüngsten Änderungen des Grundgesetzes mit Verwaltungsbezug, Entwicklungen aus dem Bereich der Digitalisierung von Verwaltung und Justiz sowie aktuelle Rechtsprechung.

"wertvolles Lehrbuch im besten Sinne"

Prof. Dr. Gernot Dörr, RV aktuell 2018, 137 zur Vorauflage

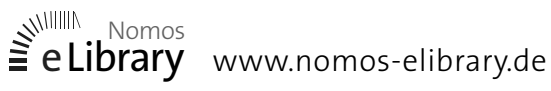

Erhältlich im Buchhandel oder

versandkostenfrei unter www.nomos-shop.de

Alle Preise inkl. Mehrwertsteuer

Xomos 